\title{
What we have learned and what the future holds: Perspectives on the COVID-19
}

\author{
Adrián Camacho-Ortiz* \\ Head of Hospital Epidemiology and Infectious Diseases, Hospital Universitario Dr. José Eleuterio González, Universidad Autónoma de Nuevo León, \\ Monterrey, Nuevo León, Mexico
}

For nearly 2 years, we have seen remarkable advances in technology and public health. From basic science to global health, the COVID-19 pandemic has put humanity to the test.

Scientists have described genomic sequences and severe acute respiratory syndrome coronavirus 2 (SARSCoV-2) virus mutations at extraordinary speed. This unprecedented progress has paved the way for the development of COVID-19 vaccines. Vaccines roll-out is greatly advancing, although with discrepancies between nations and regions, and even if most adults receive vaccination, this does not mean the virus will go away. We are simply moving to a new phase of the pandemic.

Based on scientific knowledge, we evolved our use of personal protective equipment from a full overall, fully masked, and double glove attire to a simple respirator with eye protection and a simple gown. We shifted from extreme surface disinfection practices to regular cleaning and disinfection. We learned transmission patterns and adapted our practices to reduce viral spread. Our knowledge of viral behavior aided in stopping patient retesting for proof of cure and also reduced time and costs by changing the isolation period from 14 days to 7-10 days, reactivating patients, exposed individuals, and society greatly.

\section{Will the pandemic ever end?}

The correct answer is that eventually, the pandemic status will end, giving way to regional and local outbreaks and subsequently to endemnicity and stational activity; this gradual diminishing in cases will be due to an array of factors, including immunity acquired by vaccination and/or natural immunity after the disease, part of the population that succumbed to the disease and effective treatment option in the early stages of the disease.

SARS-CoV-2 stands out compared to other coronaviruses that cause the common cold (HCoV-229E, HCoVHKU1, HCoV-NL63, and HCoV-OC43), in its greater virulence, and compared to the SARS-CoV and Middle East respiratory syndrome coronavirus (MERS-CoV) in its greater capacity for asymptomatic transmission'.

\section{The lack of an antiviral drug}

Advances in treatment for COVID-19 have been palpable. For high-risk individuals, monoclonal antibodies during the initial phase of the disease have reduced progression to hospitalization, but realistically, these are mostly unavailable for public use. Treatment of patients with supplemental oxygen requirements has evoked the interest of countless research studies, many of them with potentially effective drugs that are being analyzed. A bunch of antivirals have been tested for COVID-19 and COVID-19 pneumonia. One of the most promising was remdesivir that initially captivated the world's eyes in the ACT-I and ACT-II trials showing moderate effect in mild to moderate patients. Since then, an array of information, including real-world data, has emerged

\section{Correspondence:}

*Adrián Camacho-Ortiz

E-mail: acamacho_md@yahoo.com
Available online: 01-12-2021

Date of reception: 19-10-2021

Date of acceptance: 26-10-2021 DOI: 10.24875/RMU.M21000063
Medicina Universitaria. 2021;23(4):107-109

www.medicinauniversitaria.org license (http://creativecommons.org/licenses/by-nc-nd/4.0/). 
showing no real benefit when remdesivir is administered. Recently the molnupiravir trial was stopped early because of the clear benefit in patients receiving the drug. The interim analysis showed a more than $50 \%$ reduction in hospitalization when patients received the drug in the early stages of the disease; the pharmaceutical company has now submitted their data for Food and Drug Administration (FDA) approval. Will this or other drugs be the key element to reducing mortality.

\section{Vaccination as the cornerstone}

As of October $18,2021,47.6 \%$ of the world population has received at least one dose of a COVID-19 vaccine. Currently, 6.67 billion doses have been administered globally, and 19.23 million are now administered each day. Only $2.7 \%$ of people in low-income countries have received at least one dose ${ }^{2}$. To date there are 19 different vaccines have been administered to people around the world, of these, five vaccines are the most widely administered in the world; (Pfizer/BioNTech ${ }^{\circledR}$, Oxford-AstraZeneca ${ }^{\circledR}$, Moderna $^{\circledR}$, Janssen $^{\circledR}$, Sputnik ${ }^{\circledR}$, and Coronavac ${ }^{\circledR}$.

We now know that some combinations of vaccines give a robust immunological response, such as AstraZeneca followed by Pfizer-BioNTech, and every day new information regarding heterologous vaccination (using subsequent vaccines of another mechanism of delivery for bootstring immunity) emerges, paving the way for safe and effective universal access to vaccines.

Although when it comes to vaccines, "mixing and matching" is not something new. In many regions, vaccine roll-out has been inconsistent and second dosing has been delayed and sometimes has been mixed with different vaccines because of shortages. Some groups are now studying this, although no severe adverse events have been noted when giving doses of heterologous vaccines.

The FDA plans to allow people to receive a different vaccine as a booster than the one they initially received. This will provide flexibility to doctors and other vaccinators. The recommendation will be to use the same vaccine whenever possible, but vaccine providers could use their discretion to offer a different brand ${ }^{3}$.

\section{Misinformation spreads faster and more aggressively than any virus}

One of the most important barriers to the control of the pandemic has been misinformation and fake news.
These inaccurate data have been spread not only by the population but also by politicians, actors, singers, and preachers, among others. In Brazil, it resulted in indigenous populations greeting public health officials with bows and arrows. Misinformation in India incentivized the public to use ineffective treatments and homeopathic medications and remedies not backed by scientific evidence demonstrating efficacy in COVID-19. In Mexico, the Institute of Social Security gave away COVID-19 treatment kits that included ivermectin as therapy, potentially endangering hundreds of thousands of patients.

A Russian disinformation campaign promoting fake news made it harder for the EU itself to implement its pandemic response ${ }^{4}$. Groups of anti-vaccine advocates grew strong in the face of new vaccine technologies and their approval for emergency use. These groups spread uneasiness among the population. We need to learn from these experiences to communicate scientific knowledge better and minimize the proliferation of false data ${ }^{5}$.

\section{Social media was and will be a key element}

Messaging applications such as WhatsApp ${ }^{\circledR}$, Tele$\operatorname{gram}^{\circledR}$, Messenger ${ }^{\circledR}$, aided family and friends to keep connected, but also helped the medical community enormously to communicate adequately without personal physical contact. For the $1^{\text {st }}$ time in history, the world population had a minute by minute update on the global pandemic data at their hands; social media such as Twitter $^{\circledR}$, YouTube ${ }^{\circledR}$, Instagram ${ }^{\circledR}$, and Facebook $^{\circledR}$ provided their users with up to the minute numbers regarding confirmed cases, mortality, hospitalization, vaccination roll-out, etc. These also helped the patient keep in touch with their doctor or medical staff to monitor symptoms and overall health statuses. Through these means, science must follow the same patterns for the information to be reached in a prompt and precise matter.

The coming months will be decisive; global vaccination is the cornerstone for controlling the pandemic; regulatory agencies have to be flexible, providing the legal means by which vaccination goals can be reached. Approval of a wide variety of vaccination brands for emergency use and mix and matching will give much-needed guidance. Availability of effective antiviral drugs will undoubtedly be a game-changer for countries and regions that can afford them; emphasis should be 
placed on availability if they are this game-changer for humanity.

\section{Funding}

There are no financial disclosures to declare.

\section{Conflicts of interest}

There are no conflicts of interest to declare.

\section{References}

1. Telenti A, Arvin A, Corey L, Corti D, Diamond MS, García-Sastre A, et al. After the pandemic: perspectives on the future trajectory of COVID-19. Nature. 2021;596:495-504

2. Mathieu E, Ritchie H, Ortiz-Ospina E, Roser M, Hasell J, Appel C, et al. A global database of COVID-19 vaccinations. Nat Hum Behav. 2021;5:947-53.

3. Available from: https://www.nytimes.com/2021/10/18/us/politics/fda-mixand-match-boosters.html [Last accessed on 2021 Oct 19].

4. Kavanagh KT, Pontus C, Pare J, Cormier LE. COVID-19 lessons learned a global perspective. Antimicrob Resist Infect Control. 2021;10:125.

5. Skegg D, Gluckman $P$, Boulton G, Hackmann H, Karim SSA, Piot P Woopen C. Future scenarios for the COVID-19 pandemic. Lancet. 2021;397:777-8 